\title{
Green gas for grid as an eco-friendly alternative insulation gas to $\mathrm{SF}_{6}$ : From the perspective of PD initiated by metallic particles under DC
}

\author{
Guoming Wang ${ }^{1}$, Jiahua Shen ${ }^{1}$, \\ Demao Liu ${ }^{1}$, Sung-Wook Kim² ${ }^{2}$ Gyung-Suk Kil ${ }^{3}$
}

\begin{abstract}
This paper dealt with characteristics of partial discharge (PD) initiated by metallic particles under DC voltage in green gas for grid (g3), which is an emerging and promising eco-friendly alternative insulation gas to $\mathrm{SF}_{6}$. Experimental setup was configured to simulate PD under DC in gas-insulated power facilities. Two types of particle, namely rectangle particle and sphere particle were used. The results indicated that the discharge inception voltages in g3 gas were 90.1-92.5\% of that in $\mathrm{SF}_{6}$. In two particles, $\mathrm{PD}$ occurred with higher average apparent charge and discharge repetition rate in g3 compared with those in $S F_{6}$ gas. The time-resolved partial discharge presented similar characteristics in g3 and $\mathrm{SF}_{6}$ gas. The time interval between two successive discharge pulses were $0.1 \mathrm{~ms}-10 \mathrm{~ms}$ and $5 \mathrm{~ms}-15 \mathrm{~ms}$ in the rectangle particle and sphere particle, respectively. Results from this paper are expected to provide fundamental material for the development of $S F_{6}$ -free gas-insulated power apparatus.
\end{abstract}

K e y w o r d s: gas-insulated power facilities, green gas for grid (g3), $S F_{6}$ alternative, partial discharge, metallic particle

\section{Introduction}

Since 1960s, Sulfur hexafluoride $\left(\mathrm{SF}_{6}\right)$ has been used as the most common insulation gas in the gas-insulated power facilities, such as the gas-insulated switchgear (GIS), gas-insulated circuit breaker (GCB), and gasinsulated transmission line (GIL) owing to its high dielectric strength, excellent arc-quenching ability, and good heat transfer ability [1-4]. The use of $\mathrm{SF}_{6}$ permits the compact dimension and reliable operation of power apparatus. However, $\mathrm{SF}_{6}$ is one of the six greenhouse gases indicated by Intergovernmental Panel on Climate Change (IPCC) [5]. It has a global warming potential (GWP) of 23,500 times of that of carbon dioxide $\left(\mathrm{CO}_{2}\right)$ and a long lifetime of 3200 years that is more than 33 time longer than that of $\mathrm{CO}_{2}$. It was reported that $1 \mathrm{~kg}$ of $\mathrm{SF}_{6}$ released into the atmosphere has the equivalent global warming impact as 23.5 tons of $\mathrm{CO}_{2}$ [6]. Therefore, use and emission of $\mathrm{SF}_{6}$ are being regulated, putting the investigation of an eco-friendly alternative insulation gas to $\mathrm{SF}_{6}$ on the agenda. Although possible candidates including dry air [7], Nireogen $\left(\mathrm{N}_{2}\right)$ mixtures [8,9], perfluorocarbons [1], trifluoroiodomethane $\left(\mathrm{CF}_{3} \mathrm{I}\right)[10,11]$, hydrofluoroolefins $[1,12]$ have been studied, all of them were verified not appropriate for high voltage insulation application owing to low dielectric strength, carcinogenicity, mutagenicity, and low liquefaction temperature.

Until now, green gas for grid (g3, trademark of General Electric Company), which is a gas mixture composed of NOVECTM 4710 and $\mathrm{CO}_{2}$, has been regarded as the most promising alternative to $\mathrm{SF}_{6}$ [13]. It was investigated that $\mathrm{g} 3$ gas provides an ideal compromise between insulation performance and minimum operating temperature of power facilities, while significantly reduces the environmental concerns. The AC withstand voltage measured on a $145 \mathrm{kV}$ GIS at atmospheric pressure showed that g3 gas with 18-20\% NOVECTM 4710 has dielectric strength equivalent to $\mathrm{SF}_{6}[7,14,15]$. AC breakdown voltage tested in homogeneous, quasi- homogeneous, and non-uniform electrode configurations presented that $20 \%$ NOVECTM $4710 / 80 \% \mathrm{CO}_{2}$ is an optimal potential substitute to $\mathrm{SF}_{6}$ in medium-voltage switchgear [16]. Lightning impulse breakdown voltage carried out in different electrode arrangements indicated that $0.88 \mathrm{MPa}$ and 1.04MPa $3.7 \%$ NOVECTM $4710 / 96.3 \% \mathrm{CO}_{2}$ have the same insulation performance to $0.55 \mathrm{MPa}$ and $0.65 \mathrm{MPa}$ $\mathrm{SF}_{6}$, respectively. The liquefaction temperature of 0.88 $\mathrm{MPa}$ gas is $-30^{\circ} \mathrm{C}$, meeting the required minimum ambient operating temperature of outdoor switchgear $[17,18]$. As for environmental concerns, NOVECTM 4710 has a GWP of 2100 and an atmospheric lifetime of 30 years, both of which are much less than those of $\mathrm{SF}_{6}$. The ozone depletion potential is zero $[19,20]$. The $4 \%$ NOVECTM $4710 / 96 \% \mathrm{CO}_{2}$ has a GWP of 378 , which is reduced by $98.4 \%$ of $\mathrm{SF}_{6}$ [17]. In addition, g3 gas has required properties for electrical insulation application, including low toxicity, non-flammable, compatibility with other materials, high heat stability, and excellent switching performance [21-24]. Although insulation performances of g3 gas were investigated in terms of $\mathrm{AC}$ breakdown volt-

${ }^{1}$ Hangzhou Guozhou Power Technology Co., Ltd., Hangzhou 310015, China, guoming-wang@hotmail.com, ${ }^{2}$ College of Engineering, Silla University, Busan 46958, Korea, number1@silla.ac.kr, ${ }^{3}$ Department of Electrical and Electronics Engineering, Korea Maritime and Ocean University, Busan 49112, Korea, kilgs@kmou.ac.kr 


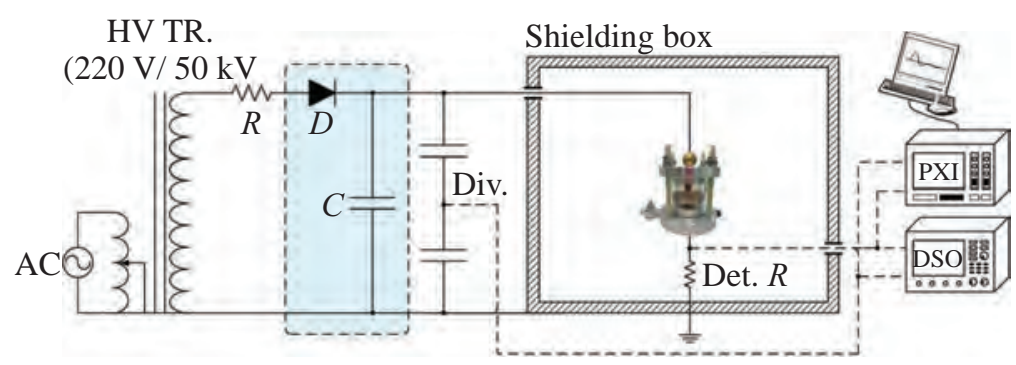

Fig. 1. experimental setup

age and lightning impulse breakdown voltage, few studies have been carried out to deal with its partial discharge (PD) characteristics under DC voltage. PD causes progressive deterioration of insulation system and is an early stage and an indicator of insulation breakdown, and it is therefore an essential part to evaluate the insulation ability of newly developed g3 gas [25-28].

In gas-insulated power apparatus, free moving metallic particles are the most common insulation defects that generated during the process of production, assembly, transportation, and switching operation [28,29]. Such particles can move along the enclosure, jump and finally reach the high voltage conductor, or attach on the insulation spacer, leading to insulation breakdown or insulator flashover [31, 32]. Therefore, free particles (FP) were selected in this paper to compare the $\mathrm{PD}$ characteristics in $\mathrm{SF}_{6}$ and $\mathrm{g} 3$ under DC, for the purpose of investigating the availability in using g3 to replace $\mathrm{SF}_{6}$. PD characteristics in terms of discharge inception voltage (DIV), apparent charge, discharge repetition rate, and time-resolved partial discharge (TRPD) characteristics were analyzed.

\section{Experiment and method}

The configuration of experimental setup is illustrated in Fig. 1. A 220V/50kV dry-type transformer controlled by a regulating transformer was used to apply high voltage AC. For generating high voltage DC, a rectifying diode and a capacitor were used. A resistor was connected in series with the circuit to limit the current in the test system. The transformer, resistor and diode were immersed in insulation oil to avoid corona occurring adjacent the connection terminal. A capacitive high voltage probe with a ratio of 10 000:1 was used to measure the applied voltage. An electrode system was fabricated to generate PD initiated by FP. For accuracy PD measurement, a non-inductive detection resistor was connected between the lower plane of electrode and ground to detect discharge current pulse. The electrode system and detection resistor were placed in a shielding room to reduce external interface. Signal acquisition was achieved by using a digital storage oscilloscope (DSO) with a samplingrate of $5 \mathrm{GS} / \mathrm{s}$ and NI PXI with a sampling rate of $250 \mathrm{MS} / \mathrm{s}[33,34]$.
Figure 2(a) shows the electrode system that was used for simulating PD initiated by FP in gas-insulated structures. A sphere electrode with a curvature radius of 10 $\mathrm{mm}$ was used as the high voltage conductor and a concave plane electrode was grounded to present the enclosure of gas-insulated structures. The distance between two electrodes was $10 \mathrm{~mm}$. Two types of particles, including the sphere particle with a diameter of $5 \mathrm{~mm}$ and the rectangle particle with a size of $15 \mathrm{~mm}$ (length) $\times 5 \mathrm{~mm}$ (width), were used to simulate the free moving insulation defects. Types of metallic particles are shown in Fig. 2(b), both of which were collected from the manufacturing site and may appear in gas-insulated structure. The electrode systems were filled with $\mathrm{SF}_{6}$ gas or g3 gas with $4 \%$ NOVECTM 4710/96 \% $\mathrm{CO}_{2}$. Before each experiment, the electrode system was vacuumed for 30 minutes by a vacuum pump and then was filled with $\mathrm{CO}_{2}$ and vacuumed again. This procedure was repeated 3 times in order to prevent any pollution of gas. In addition, the electrodes were conditioned with metal polishing paste and were cleaned carefully with isopropyl alcohol [15-17].

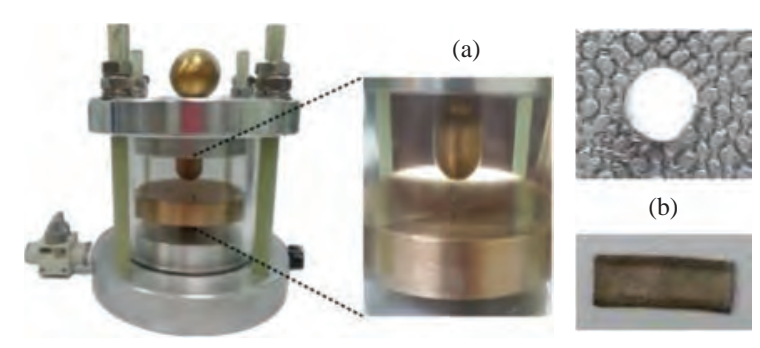

Fig. 2. experimental setup: (a)- electrode system, (b) - particle

The DIV in this paper was defined as the applied voltage at which repetitive $\mathrm{PD}$ pulses with magnitude over 10 picocoulomb $(\mathrm{pC})$ were first observed when the voltage applied to electrode system was increased gradually [32]. Since FP initiated PD has no phase correlation and DC voltage has no phase information, the TRPD method rather than phase-resolved partial discharge was used to analyze discharge pulse sequences. Characteristics including relation between discharge magnitude and time interval to preceding pulse $q\left(\Delta t_{\text {pre }}\right)$, relation between discharge magnitude and time interval to successive 


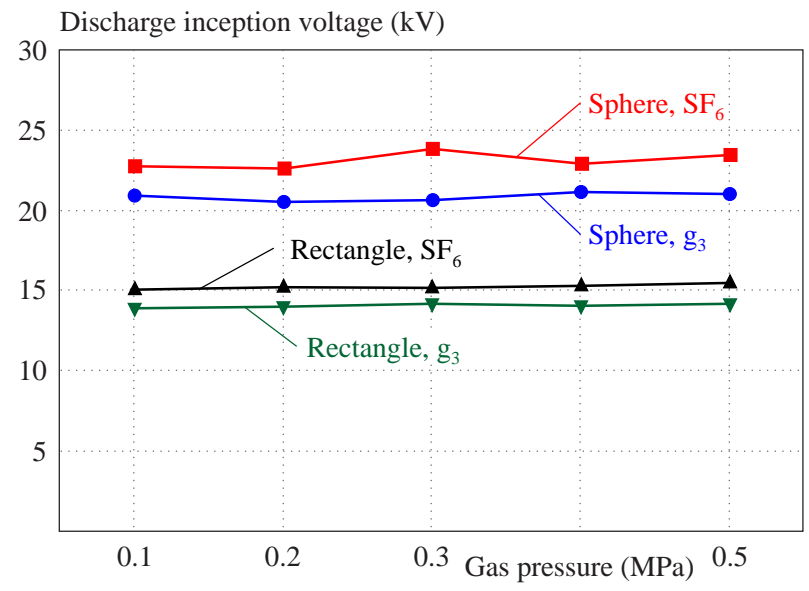

Fig. 3. discharge inception voltage depending on gas pressure

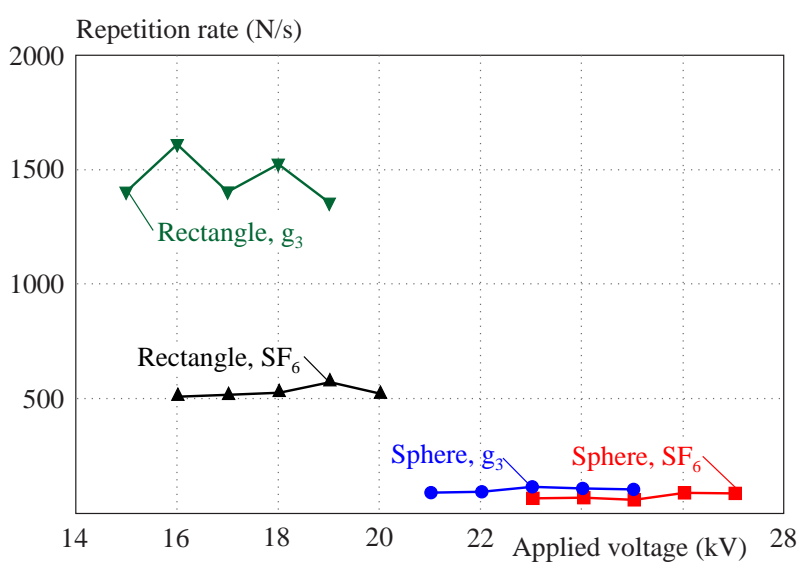

Fig. 5. discharge repetition rate depending applied voltage in $\mathrm{SF}_{6}$ and g3 gasand

pulse $q\left(\Delta t_{\text {suc }}\right)$, density function of the discharge magnitude $H(q)$, and density function of discharge time interval $H(\Delta t)$ were extracted from TRPD. The analysis application was developed based on LabVIEW real-time operation system that captured high-frequency PD pulse with precise timing and high degree of reliability.

\section{Results and discussion}

\section{1 discharge inception voltage}

Figure 3 shows the DIVs in $\mathrm{SF}_{6}$ and g3 gas with $4 \%$ NOVECTM $4710 / 96 \% \mathrm{CO}_{2}$ of two types of defects depending on the gas pressure. In each specific case, the DIVs did not change greatly with the gas pressure as PD occurred only when the particle moved owing to the coulombic force. The DIVs of rectangle particle were much lower than those of sphere particle. This was because the electric filed concentrated more intensively at the sharp edge of rectangle particle, making PD occur at a lower voltage level. The average DIVs of rectangle particle in $\mathrm{SF}_{6}$ and g3 gas were $15.3 \mathrm{kV}$ and $14.1 \mathrm{kV}$, respectively. In the sphere particle, the DIVs of g3 gas were $90.1 \%$ of that of $\mathrm{SF}_{6}$ gas, which were $20.9 \mathrm{kV}$ and 23.2 $\mathrm{kV}$, respectively.

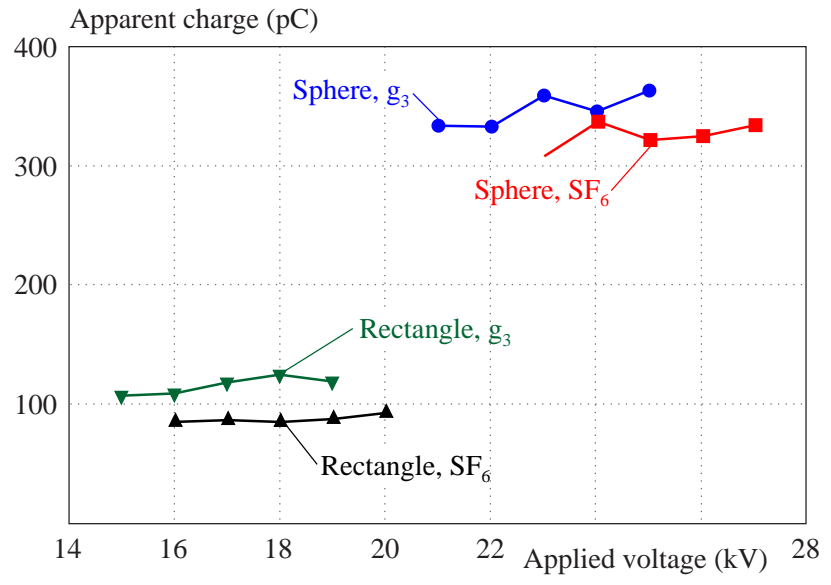

Fig. 4. Average apparent charge depending applied voltage in $\mathrm{SF}_{6}$ and g3 gas

\section{2 apparent charge and discharge repetition rate}

The change of average apparent charge with the applied voltage of two particles in $\mathrm{SF}_{6}$ and g3 is demonstrated in Fig. 4. As the applied voltage increased, the average apparent charge increased slightly. In the rectangle particle, the average apparent charge at different voltages in $\mathrm{SF}_{6}$ gas was $87 \mathrm{pC}$ whereas it was $116 \mathrm{pC}$ in g3 gas. The average apparent charges of sphere particle were $320 \mathrm{pC}$ and $347 \mathrm{pC}$ in $\mathrm{SF}_{6}$ and g3 gas, respectively. It was revealed that the apparent charge in g3 gas is higher than that in $\mathrm{SF}_{6}$. The discharge repetition rate depending applied voltage in $\mathrm{SF}_{6}$ and g3 gas is shown is Fig. 5. The average repetition rate of rectangle particle in $\mathrm{SF}_{6}$ and g3 were $531 \mathrm{~N} / \mathrm{s}$ and $1466 \mathrm{~N} / \mathrm{s}$, respectively. In the sphere particle, the average discharge repetition rate was $81 \mathrm{~N} / \mathrm{s}$ in $\mathrm{SF}_{6}$ and $104 \mathrm{~N} / \mathrm{s}$ in g3. Since higher voltage was applied to the sphere particle, PD presented greater apparent charge in the sphere particle compared with the rectangle particle. Owing to more intensive electric field concentration, discharge repetition rate in the rectangle particle is higher than that in the sphere particle.

\subsection{Time-resolved partial discharge (TRPD) character- istics}

The TRPD characteristics presented similar distribution regardless of the types of insulation gas. Figure 6 and Fig. 7 show examples of TRPD characteristics of rectangle particle in $\mathrm{SF}_{6}$ and g3 gas, respectively, including $q\left(\Delta t_{\text {pre }}\right), q\left(\Delta t_{\text {suc }}\right), H(q)$, and $H(\Delta t)$. Most of PD pulses had apparent charge lower than $200 \mathrm{pC}$ and the time interval between two successive pulses distributed in $0.1 \mathrm{~ms}-10 \mathrm{~ms}$. In sphere particle, most of PD pulses occurred with magnitude lower than $500 \mathrm{pC}$ and the time interval between two successive pulses had a range from 5m-15 ms. 


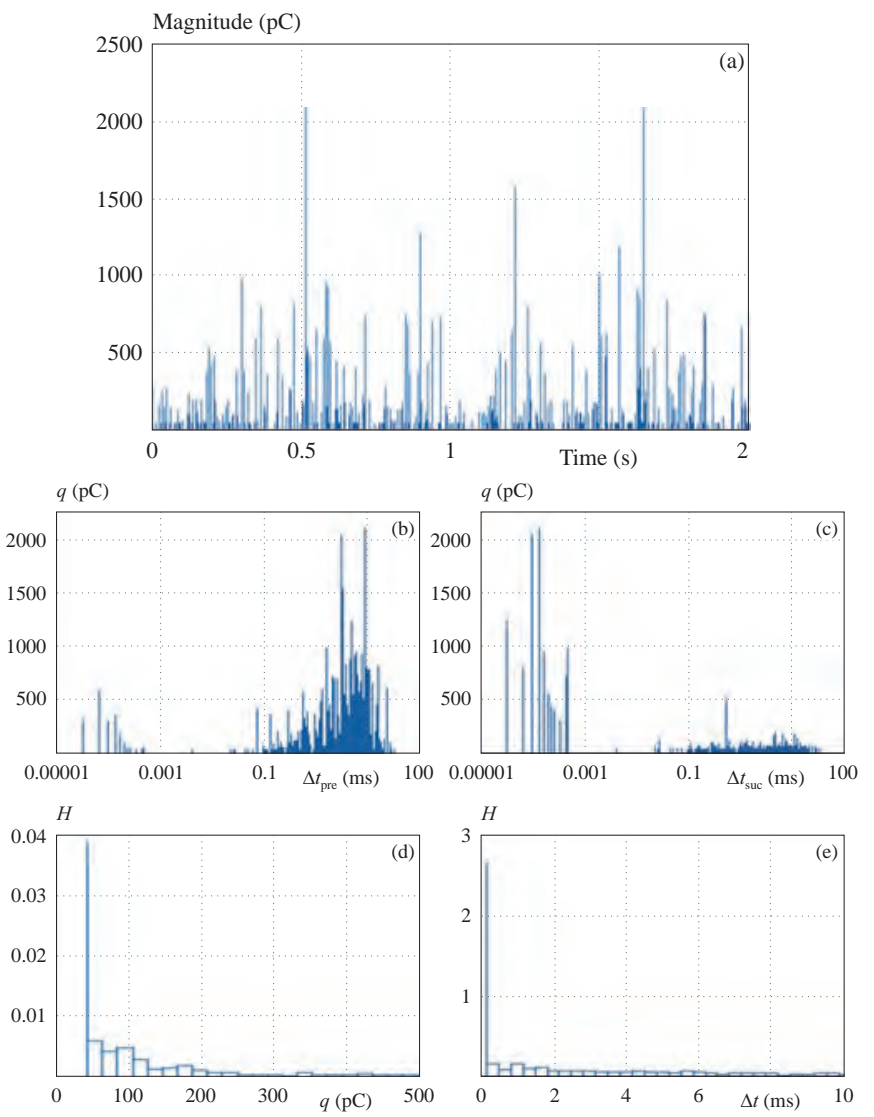

Fig. 6. TRPD characteristics of sphere particle in $\mathrm{SF}_{6}:(\mathrm{a})-\mathrm{TRPD},(\mathrm{b})-q\left(\Delta t_{\mathrm{pre}}\right),(\mathrm{c})-q\left(\Delta t_{\mathrm{suc}}\right),(\mathrm{d})-H(q)$, and $(\mathrm{e})-H(\Delta t) /$
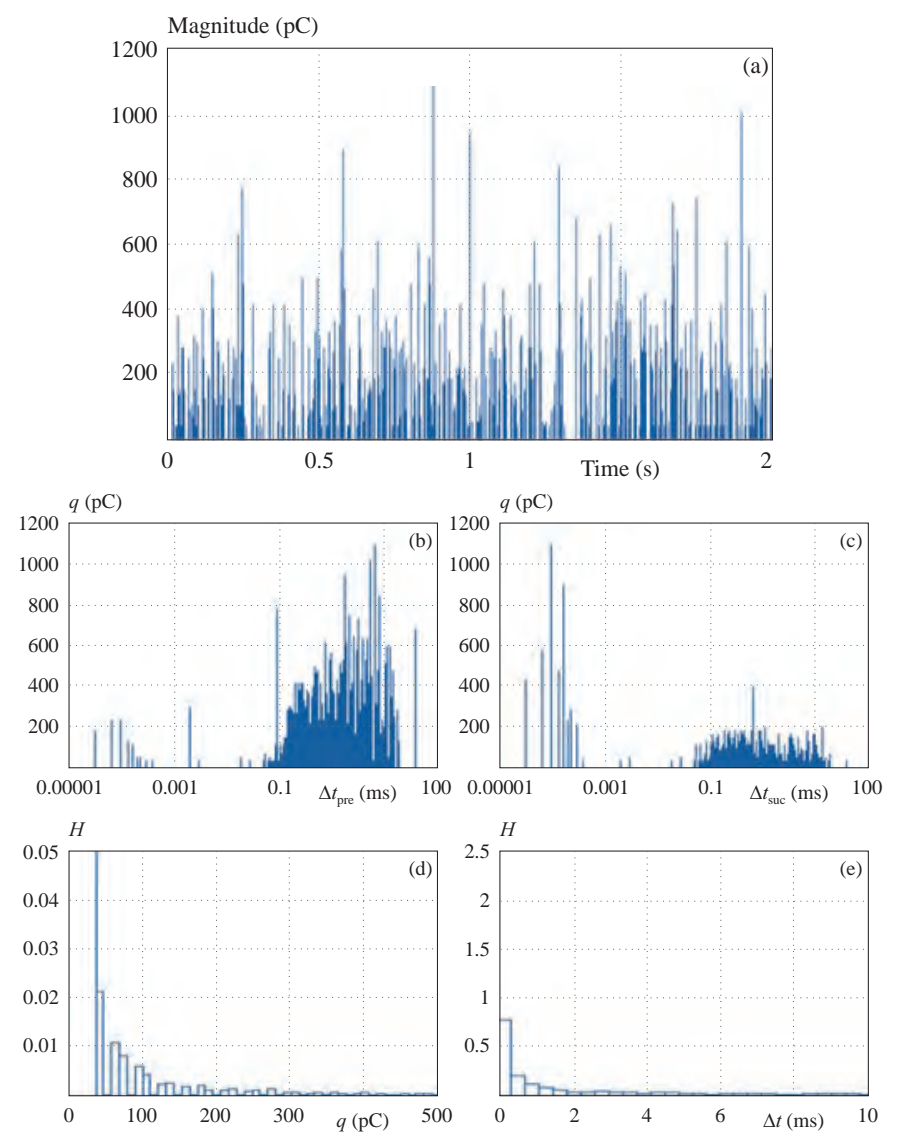

Fig. 7. TRPD characteristics of sphere particle in g3: (a) - TRPD, (b) $-q\left(\delta t_{\text {pre }}\right),(\mathrm{c})-q\left(\delta t_{\text {suc }}\right),(\mathrm{d})-H(q)$, and (e) $-H(\delta t)$ 


\section{Conclusions}

In this paper, $\mathrm{PD}$ initiated by metallic particles under DC voltage was studied in $\mathrm{SF}_{6}$ and g3 gas for investigating the performance of promising eco-friendly insulation gas alternative to $\mathrm{SF}_{6}$. PD characteristics, namely DIV, apparent charge, discharge repetition rate, and TRPD characteristics were analyzed. From the results, the DIVs in g3 gas were 90.1-92.5\% of that in $\mathrm{SF}_{6}$ depending on the types of particles. In addition, both the average apparent charge and the discharge repetition rate in g3 were higher than those in $\mathrm{SF}_{6}$ gas. The TRPD characteristics distributed with similar patterns regardless of the type of insulation. Although g3 gas showed slightly lower insulation performance compared with $\mathrm{SF}_{6}$, it is regarded as the most promising eco-friendly insulation gas to be applied in the gas-insulated power facilities given the environmental concerns. Results from this paper are expected to be used for insulation design of $\mathrm{SF}_{6}$-free gas-insulated electrical apparatus using eco-friendly g3 gas.

\section{REFERENCES}

[1] A. Beroual and A. Haddad, "Recent Advances in the Quest for a New Insulation Gas with a Low Impact on the Environment to Replace Sulfur Hexafluoride (SF6) gas in High-voltage Power Network Applications, Energies, vol. 10, no. 8, pp. 1216, 2017.

[2] M. G. Danikas and F. V. Topalis, "Partial Discharge Considerations in Gas Insulated Switchgear (GIS)", Journal of Electrical Engineering, vol. 53, no. 9, pp. 281-284, 2000.

[3] M. G. Danikas and F. V. Topalis, "[", A, Haddad D, F, Warne Advances in high voltage engineering, 1st ed, United Kingdom: The Institution of Engineering Technology, pp. 37-73, 2004.

[4] S. Xiao, X. Zhang, J. Tang, and S. Liu, "A Review on SF6Substitute Gases Research Status of CF3I Gases", Energy Report vol, 4, pp. 486-496, 2018.

[5] S. Xiao, X. Zhang, J. Tang, and S. Liu, "[", Intergovernmental Panel on Climate Change Guidelines fornational greenhouse gas inventories, 2006.

[6] Y. Kieffel, F. Biquez, and P. Ponchon, "Alternative Gas to SF6 for use in High Voltage Switchgears: g3", International Conference on Electricity Distribution, pp. 1-5, 2015.

[7] H. Okubo and A. Beroual, "Recent Trend Future Perspectives in Electrical Insulation Techniques in Relation to Sulfur Hexafluoride (SF6) Substitutes for High Voltage Electric Power Equipment", IEEE Electrical Insulation Magazine, vol. 27, no. 2, pp. 34-42, 2011.

[8] T. Rokunohe, Y. Yagihashi, F. Endo, and T. Oomori, "FundamentalInsulationCharacteristics of air, N2, CO2, N2/O2", Electrical Engineering in Japan, vol. 155, no. 3, pp. 9-17, 2006.

[9] H. Goshima, H. Shinkai, and M. yashima, "Lightning Impulse Breakdown Characteristics of High-Pressure N2as an AlternativeInsulationGas to SF6", Gaseous Dielectrics IX, pp. 359-364, 2001.

[10] L. Chen, P. Widger, M. S. Kamarudin, H. Griffiths, and A. Haddad, "CF3I Gas Mixtures: Breakdown Characteristics Potential for Electrical Insulation", IEEE Transactions on Power Delivery 32, no, 2, pp. 1089-1097, 2017.

[11] X. Zhang, S. Xiao, J. Zhou, and J. Tang, "Experimental Analysis of the Feasibility of CF3I/CO2 Substituting SF6 asInsulation Medium using Needle-plate Electrodes", IEEE Transactions on Dielectrics Electrical Insulation, vol. 21, no. 4, pp. 1895-1900, 2014.
[12] A. Chachereau, M. Rabie, and C. M. Franck, "Electron Swarm Parameters of the Hydrofluoroolefine HFO1234ze", Plasma Sources Science Technology, vol. 25, no. 4, pp. 045005, 2016.

[13] "GE Grid Solutions SF6 Alternative For High Voltage Applications", Available online: https://www.gegridsolutions.com/HV MV_Equipment /catalog/g3/(accessed on December 2019).

14] Y. Kieffel, T. Irwin, P. Ponchon J. Owens, "Green Gas to Replace SF6 in Electrical Grids", IEEE Power Energy Magazine, vol. 14, no. 2, pp. 32-39, 2016.

15] G. M. Wang, W. H. Kim, G. S. Kil, S. W. Kim, and J. R. Jung, "Green Gas for a Grid as An Eco-Friendly Alternative Insulation Gas to SF6: From the Perspective of Partial Discharge Under AC", Applied Science, vol. 9, no. 4, pp. 651, 2019.

16] B. Zhang, N. Uzelac Y. Cao, "Fluoronitrile/CO2 Mixture as an Eco-friendly Alternative to SF6 for Medium Voltage Switchgears", IEEE Transactions on Dielectrics Electrical Insulation, vol. 25, no. 4, pp. 1340-1350, 2018.

17] H. E. Nechmi a Beroual, A. Girodet, and P. Vinson, "Fluoronitriles /CO2 Gas Mixture as Promising Substitute to SF6 for Insulation in High Voltage Applications", IEEE Transactions on Dielectrics Electrical Insulation, vol. 23, no. 5, pp. 2587-2593, 2016.

[18] Institute of Electrical Electronics Engineers IEEE C37, 100, 1-IEEE standard of common requirements for high voltage power switchgear rated above $1000 \mathrm{~V}$, 1st ed, USA: IEEE, 2007,.

[19] $3 \mathrm{M}^{\mathrm{TM}}$ Novec $^{\mathrm{TM}} 4710$ Insulating Gas, pp. 1-4, 2017.

[20] Y. Tu, Y. Cheng, C. Wang, X. Ai, F. Zhou, and G. Chen, "Insulation characteristics of Fluoronitrile/ $\mathrm{CO}_{2}$ Gas Mixture under DC Electric Field", IEEE Transactions on Dielectrics Electrical Insulation, vol. 25, no. 4, pp. 1324-1311, 2018.

21] Y. Kieffel, F. Biquez, D. Vigouroux, P. Ponchon, A. Schlernitzauer, R. Magous, G. Cros, and J. G. Owens, "Characteristics of g3 an Alternative to SF6", International Conference \& Exhibition on Electricity Distribution, pp. 54-57, 2017.

[22] Y. Kieffel, A. Girodet, F. Biquez, P. Ponchon, J. Owens, M. Costello, M. Bulinski, R. V. San, and K. Werner, "SF6 Alternative Development for High Voltage Switchgears", CIGRE 2014, pp. 305, 2014.

23] Y. Kieffel and F. Biquez, "SF6 Alternative Development for High Voltage Switchgears", Electrical Insulation Conference, pp. 379-383, 2015.

[24] J. G. Owens, "Greenhouse Gas Emission Reductions through Use of a Sustainable Alternative to SF6", Electrical Insulation Conference, pp. 535-538, 2016

[25] W. Chang, F. Du, J. Bi, J. Shao, J. Peng, J. Liu, and H. Wang, "Development Process of Micropores Partial Discharge of Silicon Rubber in Prefabricated Cable Joint", Journal of Electrical Engineering, vol. 69, no. 5, pp. 366-372, 2018.

26] G. M. Wang, S. J. Kim, G. S. Kil, and S. W. Kim, "Optimization of Wavelet Thresholding for Partial Discharge Detection under HVDC", IEEE Transactions on Dielectrics Electrical Insulation, vol. 24, no. 1, pp. 200-208, 2017.

[27] R. Sarathi, P. D. Singh, and M. G. Danikas, "Characterization of Partial Discharges in Transformer Oil Insulation under AC DC Voltage using Acoustic Emission Technique", Journal of Electrical Engineering, vol. 58, no. 2, pp. 91-97, 2007.

[28] G. M. Wang, G. S. Kil, H. K. Ji, and J. H. Lee, "Disturbance Elimination for Partial Discharge Detection in Spacer of Gas-Insulated Switchgears", Energies, vol. 10, no. 11, pp. 1762, 2017.

29] H. Ji, C. Li, Z. Pang, G. Ma, X. Cui, W. Zhao, and J. Wang, "Influence of Tip Corona of Free Particle on PD Patterns in GIS", IEEE Transactions on Dielectrics Electrical Insulation, vol. 24, no. 1, pp. 259-267, 2017.

30] U. Schichler, W. Koltunowicz, F. Endo, K. Feser, A. Giboulet, A. Girodet, H. Hama, B. Hampton, H. G. Kranz, J. Lopez-roldan, L. Lundgaard, S. Meijer, C. Neumann, S. Okabe, J. Pearson, R. Pietsch, U. Riechert, and S. Tenbohlen, "Risk Assessment on Defects in GIS based on PD Diagnosis", IEEE Transactions on 
Dielectrics Electrical Insulation, vol. 20, no. 6, pp. 2165-2172, 2013.

[31] B. Qi, C. Li, Z. Xing, and Z. Wei, "Particle Discharge Initiated by Free Moving Metallic Particles on GIS Insulation Surface: Severity Diagnosis Assessment", IEEE Transactions on Dielectrics Electrical Insulation, vol. 21, no. 2, pp. 766-773, 2014.

[32] R. Sarathi and R. Umamaheswari, "Understanding the Partial Discharge Activity Generated due to Particle Movement in a Composite Insulation under AC Voltages", International Journal of Electrical Power \& Energy Systems, vol. 48, pp. 1-9, 2013.

[33] International Electrotechnical Commission IEC 60270- High -Voltage Test Techniques-Partial Discharge Measurements, 3rd ed, Switzerland: IEC, 2000,.

[34] G. M. Wang, H. E. Jo, S. J. Kim, S. W. Kim, and G. S. Kil, "Measurement Analysis of Partial Discharges in SF6 Gas under HVDC", Measurement, vol. 91, pp. 351-359, 2016.

Received 17 June 2019

Guoming Wang was born in Liaoning, China in 1990. He received the BEng degree from Division of Engineering, Dali University, China in 2013, the MEng degree and PhD degrees from Division of Electrical and Electronics Engineering, Korea Maritime and Ocean University, Korea in 2015 and 2019, respectively. He is currently a researcher at Hangzhou Guozhou Power Technology Co., Ltd. His research interests are in the area of high voltage engineering, eco-friendly insulation gases, arc detection, and diagnosis of power facilities.

Jiahua Shen was born in Zhejiang, China in 1985. He received the BEng degree from Hunan Institute of Science and Technology, China in 2008. He is currently a technical manager at Hangzhou Guozhou Power Technology Co., Ltd. His research interests are in the area of high voltage engineering, eco-friendly insulation gases, diagnosis of power facilities.

Demao Liu was born in Zhejiang, China in 1981. He received the BEng degree from Zhejiang Gongshang University, China in 2002. He is currently a senior engineer at Hangzhou Guozhou Power Technology Co., Ltd. His research interests are condition monitoring and diagnosis of power facilities, high voltage engineering, and asset management.

Sung-Wook Kim (Professor) was born in Busan, Korea in 1981. He received the BEng, MEng, and PhD degrees in electrical engineering and high voltage engineering from the Korea Maritime University, Korea in 2008, 2010 and 2017, respectively. He worked for R\&D center of Hyosung Corporation and was one of the members in charge of DGA project for 9 years. He is currently a professor at Silla University, Korea. His research interests are partial discharge and DGA diagnosis method of power transformers, and performance evaluation of insulation gases.

Gyung-Suk Kil (Professor) was born in Incheon, Korea in 1962. He received the BEng, MEng, and PhD degrees from Inha University, Korea in 1984, 1987, and 1996, respectively. Since then, he has been a professor at the Division of Electrical and Electronics Engineering, Korea Maritime and Ocean University. He worked as a visiting professor at Cardiff Uni68 versity in 2003. His research interests are in the area of high voltage engineering, arc detection, grounding technique, lightning protection, and diagnosis of power facilities. He is a member of the IEEE Dielectrics and Electrical Insulation Society. 